\title{
Autologous Natural Killer Cell-like CTLS
}

National Cancer Institute

\section{Source}

National Cancer Institute. Autologous Natural Killer Cell-like CTLS. NCI Thesaurus. Code C156168.

A preparation of cytotoxic T-lymphocytes (CTLS) that express natural killer (NK)-like features (nCT Ls), with potential immunomodulating and antineoplastic activities. The nCT Ls are derived from autologous lymphocytes that have been in vitro exposed to autologous alpha-type-1 polarized dendritic cells that are pulsed with specific autologous tumor-associated antigens (TAAs); the nCT Ls are subsequently expanded in the presence of the cytokine human interleukin-2 (IL-2). The generated nCT Ls are potent CT Ls that produce high amounts of granzyme B and perforin, and interferon-gamma (IFNg) with high killer activity and tumor-homing potential. Upon infusion of the autologous nCTLs, these cells specifically recognize the TAAs on the tumor cells, then bind to and directly lyse tumor cells. 